\title{
脊髓内生长抑素参与 $\mathbf{P}$ 物质引起的 伤害性信息传递
}

\author{
阮怀珍 李希成 蔡文琴(1) \\ (第三军医大学生理学教研室, (1)组胚教研室,重庆 630038)
}

\section{关键词生长抑素 $\mathrm{P}$ 物质 原位杂交 兔疫组化 举敷}

$\mathrm{P}$ 物质 (substance P, SP) 与生长抑素 (somatostatin, SOM) 共同分布于脊髓, 它们都与伤害 感受性信息传递有关. 我们以往的工作证实脊髄内 SP 主要起致痛作用, 并可加强伤害性刺 激引起的痛反应 ${ }^{[1]}$. 据报道, SOM 也是伤害性初级传入神经末梢释放的一种神经递质或神经 调制物 ${ }^{[2]}$. 但是脊䯈内 SOM 是否参与 P 物质引起的伤害感受性信息传递, 尚不清楚. 本研 究应用免疫组化技术及 $\mathrm{c}-$ fos 和 SOM 免疫组化双重染色方法研究了这两种免疫标记物的关 系, 为了对 SP 诱导 SOM 合成的可能性进行探讨, 进一步用原位杂交 (in situ hybridization) 技 术来观察 SP 引起的伤害性信息传递过程中前 SOM 原 (perprosomatostatin, PPS)mRNA 的表 达, 探讨 SOM 在脊䯣水平对 SP 引起的伤害感受性信息传递作用的影响.

\section{1 材料与方法}

\section{1 材料}

实验用 Wistar 大鼠 22 只, 体重 $200 \sim 220 \mathrm{~g}$. SP, Sigma 产品, 溶于人工脑脊液内 (Artificial cerebrospinal fluid, ACSF), pH 5.5; PPS-mRNA 原位杂交试剂盒由第二军医大学 组胚教研室提供; c-fos抗血清 $(1: 2000)$, Cambridge ResearchBiochemicals 产品; SP抗血清 $(1: 10000)$ 和 SST 抗血清 $(1: 5000)$, Sigma 产品; ABC 试剂盒, 购自 Vector 公司.

\section{2 方法}

1.2.1 实验分组 实验分 3 组: (1) 正常对照组 (10 例), 2 例动物不给任何刺激, 作为空白对 照; 4 例右后足底注射 $150 \mu \mathrm{L}$ 生理盐水, $2 \mathrm{~h} ; 4$ 例鞘内注射(脊髓蛛网膜下腔插管方法同文献 [1]) ACSF $15 \mu \mathrm{L}, 2 \mathrm{~h}$. (2) 福尔马林组 (6 例), 大鼠右后足底注射 $5 \%$ 福尔马林 $150 \mu \mathrm{L}, 2 \mathrm{~h}$.

(3) SP 组 (6 例), 鞘内注射 SP $(10 \mu \mathrm{g} / 15 \mu \mathrm{L}), 2 \mathrm{~h}$.

1.2.2 前生长抑素原 mRNA 原位杂交法 以地高辛标记的反意 cRNA 探针按文献[3]叙 述步骤进行原位杂交前、杂交和杂交后处理。抗地高辛抗体工作浓度为 $1: 1000$, 以 NBT/ $\mathrm{BCIP}(400 \mu \mathrm{g} / \mathrm{mL} / 200 \mu \mathrm{g} / \mathrm{mL})$ 反应液显色. 对照实验包括用 $2 \mu \mathrm{g} / \mathrm{mg}$ RNA 酶 A 预处理切片 $30 \mathrm{~min}$ 和用不含探针杂交缓冲液杂交, 结果均检测不到明显的杂交信号.

1995-10-19 收稿, 1996-02-07 收修改稿 
1.2.3 兔疫组化法 (1) c-fos, SP, SOM 免疫组化法: 实验毕, 按常规进行大鼠组织灌流、固 定、切片 $(40 \mu \mathrm{m})$, 然后进行 $\mathrm{ABC}$ 免疫组化染色 ${ }^{[1,4]}$. 阴性对照分别采用正常羊(或兔)血清和 PBS $(0.01 \mathrm{~mol} / \mathrm{L})$ 代替一抗, 随后的步骤完全相同, 结果表明对照切片上见不到免疫反应阳性 细胞. (2) c-fos/SOM 免疫双重染色法: 将 c-fos 抗血清染色后的切片再置入兔抗 SOM 血清孵 育 $48 \mathrm{~h}\left(4^{\mathrm{C}} \mathrm{C}\right)$, 随后依次进入生物素结合羊抗兔血清 $(1: 200)$ 和 $\mathrm{ABC}(1: 100)$ 中各孵育 $2 \sim 4 \mathrm{~h}$ (室温), 最后用 $\mathrm{DAB}-\mathrm{H}_{2} \mathrm{O}_{2}$ 液呈色, $\mathrm{SOM}$ 免疫阳性产物为棕色.

1.2.4 数据处理 在每一动物腰 3 4 节段随机抽样 4 片, 经图象分析, 作阳性细胞计数, 取均数作为该动物的 $\mathrm{c}-\mathrm{fos}$ 样免疫反应 (c-fos-like immunoreactivity, FLI) 数, SOM 样免疫反应 (SOM-like immunoreactivity, SOM-LI) 数和 PPS-mRNA 数. 经显微分光光度计检测 SP 样免 疫反应物 (SP-like immunoreactivity, SP-LI) 的吸光度. 实验数据皆用电子计算机经双样本 $t$ 检验程序 $(P D A-2)$ 进行统计学处理. 结果以均值 \pm 标准误 $(M \pm S E)$ 表示.

\section{2 结果}

\section{1 福尔马林对眷髄 SP 和 SOM 的影响}

大鼠右后足底注射福尔马林 $2 \mathrm{~h}$ 后, FLI 神经元大量出现于脊䯣右侧背角的浅表层, 另外 固有核、背角颈部、腹侧部都有许多散在的 FLI 神经元, 而对照组脊䯣 c-fos 表达很低(图 1 (a)、(b), 表 1); 脊髓 SOM-LI 与对照组的比较也显著增加 $(p<0.01$ ), 分布于脊髓各层 (图 1 (c)、(d), 表 1);PPS-mRNA 阳性标记细胞增多, 分布于 I IV 层, 与对照组的比较差异显著 ( $p$ $<0.01$, 图 1(e)、(f), 表 1); 脊髓右侧背角 SP-LI 增多, 密集分布于 I, II 层, 与对照组的比较差 异显著 $(p<0.01$, 图 1(g)、(h), 表 1).

\subsection{SP 对眷骮速内 SOM 的影响}

大鼠鞘内注射 SP, $2 \mathrm{~h}$ 后, 可见双侧脊髓背角都有 FLI, 主要在 I, II, V, VI 层. 同时, 也可 见脊髓 SOM-LI 和 PPS-mRNA 增多, 与对照组的比较均差异显著 $(p<0.01$, 图 1(i)、(j)、(k), 表 1). 采用双重免疫组化法 $(A B C)$ 研究 $c$-fos 和 SOM 在脊隨背角的共存, 结果显示, 脊䯣背 角半数神经元呈 c-fos 或 SOM 阳性反应, 其中有一部分是双标记神经元(图 1(1)), 占 FLI 总数 的 $34.7 \%$, 占 SOM-LI 总数的 $25.6 \%$.

\section{3 讨论}

已知 SP 是伤害性初级传入神经末梢释放的兴奋性递质, 在痛觉调节过程中, 脊髓内的 SP 主要起着传递痛觉信息的作用 ${ }^{[1,4]}$. 我们以往的工作证实脊髓内 SP 主要起致痛作用,并可加 强伤害性刺激引起的痛反应 ${ }^{[1]}$. 而 SOM 也是第一级伤害性传入神经末梢释放的一种神经递 质或神经调节物 ${ }^{[2]}$, 如伤害性热刺激和化学刺激可使兔脊髓背角的 SOM 释放增多 ${ }^{[5]}$. 大鼠 足底注射福尔马林后, 可激活外周感觉神经纤维, 使之释放 $\mathrm{SP}^{[4]}$, 本实验观察到大鼠足底注射 福尔马林后也可使脊髓背角 PPS-mRNA 显著增加, 表明 SOM 和 SP 一样, 与伤害感受性信息 传递有关, 伤害性刺激可使 SOM 合成加速.

据报道, SP 在机械伤害性信息的传递中起主要作用,而 SOM 在热刺激信息的传递过程中 起重要作用 ${ }^{[5]}$. 在伤害性化学刺激信息传递中 SP 和 SOM 都起作用 ${ }^{[2]}$. 我们观察到鞘内注 射 SP, 在 c-fos 表达增加的同时, SOM-LI 和 PPS-mRNA 也增加. 双重免疫组化染色可见脊髓 


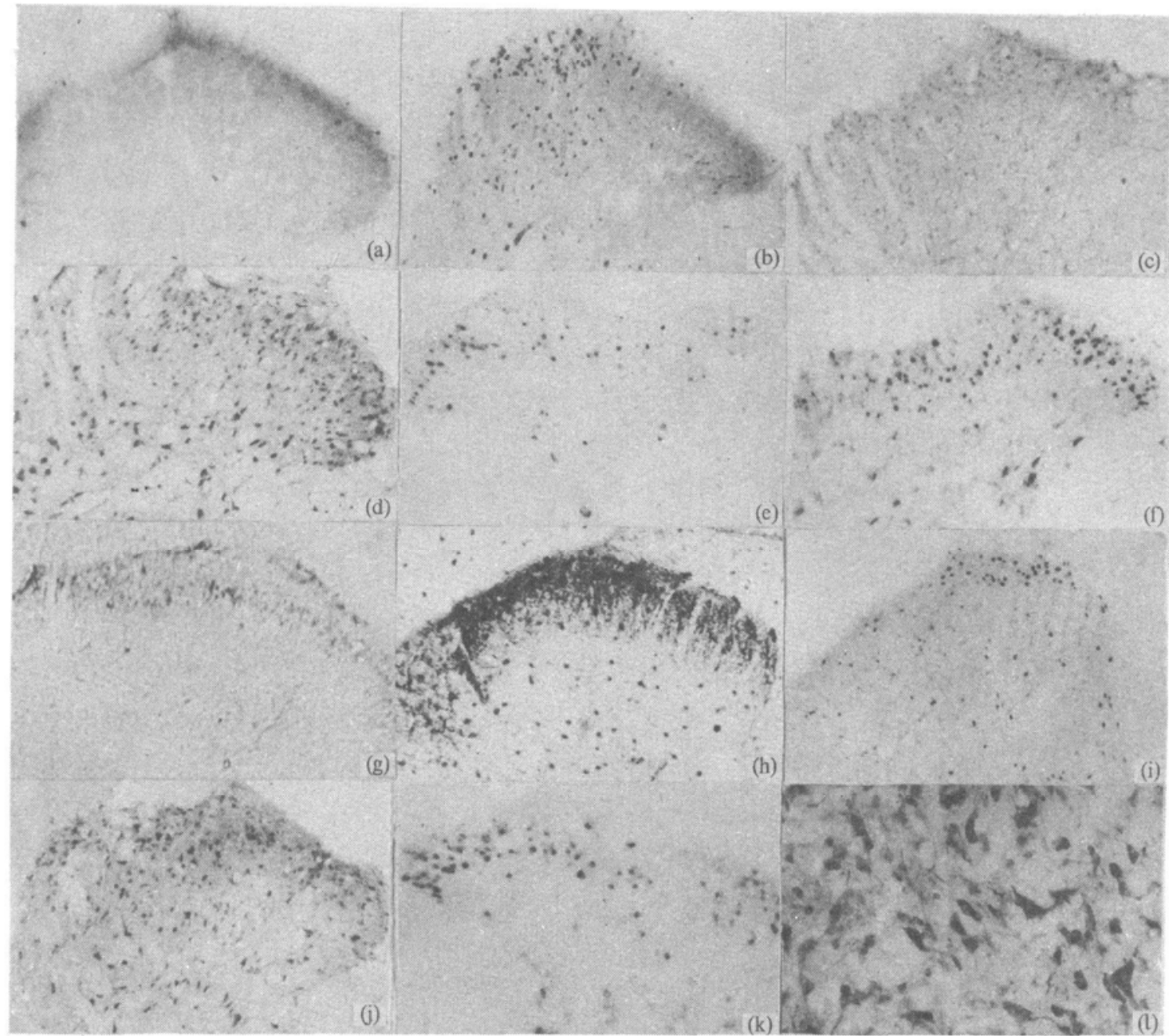

图 1 脊髓免疫组化, 原位杂交染色显微照相(右侧脊髓背角)

(a) c-fos 免疫组化染色, 足底注射生理盐水, $\times 60$; (b) c-fos 免疫组化染色, 足底注射福尔马林, 背角可见大量 FLI, $\times 60$; (c) SOM 免疫组化染色, 足底注射生理盐水, $\times 60$; (d) SOM 免疫组化染色, 足底注射福尔马林, 脊骴 背角 SOM-LI 增多, ×60; (e) Dig-SOM-cRNA 原位杂交, 足底注射生理盐水, $\times 60$; (f) Dig-SOM-cRNA 原位杂交, 足底注射福尔马林, 脊䯣背角 PPS-mRNA 增多, $\times 60 ;(\mathrm{g})$ SP 免疼组化染色, 足底注射生理盐水, $\times 72 ;(h)$ SP 免 疼组化染色, 足底注射福尔马林, 背角 SP-LI 增多, $\times 72$; (i) c-fos 免疼组化染色, 鞘内注射 SP, 背角可见大量 FLI,

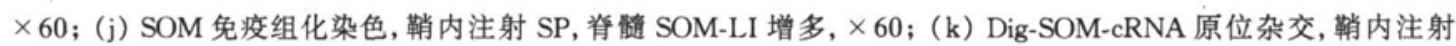
SP, 脊髓背角 PPS-mRNA 增多, $\times 60$; (1) c-fos 免疫组化与 SST 免疫组化双染色, 胞核为 FLI, 葡萄糖氧化酶-DAB-

硫酸镍铵显色, 胞质为 SOM-LI, DAB- $\mathrm{H}_{2} \mathrm{O}$ 染色, 鞘内注射 SP, $\times 240$

表 1 福尔马林及 P 物质对脊骴 SP-LI, FLI, SOM-LI 和 PPS-mRNA 的影响 ${ }^{\mathrm{a})}$

\begin{tabular}{|c|c|c|c|c|c|}
\hline 分 组 & 动物数 & SP-LI 吸光度 & FLI 数 & SOM-LI 数 & PPS-mRNA 数 \\
\hline 对 照 & 10 & $167.8 \pm 44.4$ & $4.9 \pm 0.9$ & $137.8 \pm 31.6$ & $66.5 \pm 7.6$ \\
\hline 福尔马林 & 6 & $225.7 \pm 48.6^{b)}$ & $128.8 \pm 8.1^{b)}$ & $225.7 \pm 58.4^{b)}$ & $90.7 \pm 8.4^{b)}$ \\
\hline P物质 & 6 & & $94.8 \pm 10.9^{b)}$ & $175.3 \pm 39.3^{b)}$ & $98.5 \pm 10.1^{b)}$ \\
\hline
\end{tabular}

a) $\mathrm{M} \pm \mathrm{SE} \quad$ b) $p<0.01$, 与对照组比较 
背角还有许多 FLI/SOM-LI 双标记细胞. 表明在 SP 引起的痛反应中, 内源性 SOM 与之有 关. 已知 c-fos 是一种存在于正常神经元细胞核内的原癌基因, 其可被多种刺激诱导而快速表 达 ${ }^{[6]}$. 并被称为神经元的“第三信使” ${ }^{[7]}$. 从此角度来看, 本文显示的鞘内注射 SP 后, 脊䚟背 角有许多 FLI/SOM-LI 双标记细胞, 提示 SP 可能经某种或某些途径激活脊㵦背角神经元, 诱 发 c-fos 癌基因表达, 而进一步促使 SOM 转录、合成. 由此表明, SP 可能通过 c-fos 调控脊䯠 内 SOM 的生物合成, 而产生伤害感受性信息传递作用.

\section{参考文献}

1 Ruan H Z , Li X C, Cai W Q et al. Participation of substance P in noxious stimulus-evoked c-fos expression in spinal cord. Chin Sci Bull, 1994, 39(20):1 725 1 729

2 Ohkubo T, Shibata M, Takahashi $\mathrm{H}$ et al. Role of Substance $\mathrm{P}$ and somatostatin on transmission of nociceptive information induced by formalin in spinal cord. J Pharmacol Exp Ther, 1990, 252:1 261 1268

3 蔡文琴, 王伯云等. 实用免疫细胞化学与核酸分子杂交技术, 成都: 四川科学技术出版社, 1994

4 Ruan H Z, Li X C. Effect of morphine and monoamine neurotransmtters on pain modulation of substance P in the spinal cord. Chin Sci Bull, 1991, 36(8):682 686

5 Kuraishi $Y$, Hirota N, Sato $\mathrm{Y}$ et al. Evidence that substance $\mathrm{P}$ and somatostatin transmit separate information related to pain in the spinal dorsal horn. Brain Res, 1985, 325:294 302

6 Menetrey D, Gannon A, Levine J D et al. Expression of c-fos protein in interneurons and projection neurons of the rat spinal cord in response to noxious somatic, articular, and visceral stimulation. J Comp Neurol, 1989, 285:177 195

7 Rauscher III F J, Cohen D R, Curran T et al. Fos-associated protein P39 is the product of jun pro-oncogene. Science, 1988, 240:1 010 1 016 